\title{
Energy Efficient Coded Cooperative Data Exchange for Mobile Users
}

\author{
Shahriar Etemadi Tajbakhsh and Parastoo Sadeghi \\ Research School of Engineering \\ The Australian National University \\ Canberra, 0200, Australia \\ Emails: \{shahriar.etemadi-tajbakhsh, parastoo.sadeghi\}@anu.edu.au
}

\begin{abstract}
In this paper, we generalize the problem of network coded cooperative data exchange from a fixed broadcast topology to dynamic networks with mobile peers. In this problem a group of wireless clients are interested in obtaining a set of packets through cooperation, where each client initially holds a subset of packets. Unlike recent studies where cooperation is enabled through a fixed error free broadcast channel among fixed or stationary peers, we assume that peers move randomly between transmission rounds, have a limited transmission range and suffer from packet erasures. In this case giving an exact solution to the problem of minimum number of transmissions is difficult, if not impossible. Therefore, we propose two different heuristic transmission strategies to decrease the total number of transmissions compared to uncoded transmissions. We compare the performance of these two strategies in terms of energy consumption (total number of transmissions) by analysis and simulations. In particular, we show that when packet delivery delay is not an issue, the total number of transmissions can be dramatically decreased at the price of a small overhead.
\end{abstract}

\section{INTRODUCTION}

Cooperation among the users, is one of the promising strategies in designing efficient communication networks. The notion of cooperation has been studied in many different areas of communication such as wireless physical layer [1], wireless ad hoc networks [1], [2], peer to peer networks [3], etc. The foundation of this paper is based on a recently developed topic in cooperative wireless networks called coded cooperative data exchange (CCDE) [4], [5]. In this problem, a group of wireless clients who are interested in obtaining a set of packets are considered. The set of packets is initially broadcast by a server to these clients, but for some reason each client may only receive a subset of packets. This may happen due to erasures and failures that occur in wireless links or because of clients joining in the middle of transmission. The conventional solution to this problem is retransmissions by the server. An alternative solution, recently suggested in the literature, is to cooperatively exchange the packets among clients using network coding techniques [6] once the clients hold the entire set of packets collectively. Consequently, the retransmission by the server is substituted by short range communications among clients which is faster, cheaper and more reliable and the valuable downlink bandwidth of the server is freed.

This has been the subject of recent research. In particular, [5] and [7] suggest network coding methods to reduce the total number of required transmissions and delay, respectively.
The optimal number of transmissions was later shown to be the solution to a linear program [8], [9], where in [8] a general minimum cost solution to this problem and fairness were also studied. Extending the results of [5], the authors in [10] proposed a randomized CCDE algorithm while taking the cost of transmissions into account. In randomized CCDE, clients exchange packets which are linear combinations of the packets in their possession using random coefficients from a sufficiently large finite field [11].

In this paper, for the first time, we consider the problem of CCDE in a dynamic environment, where the clients are mobile. To clarify the position of this paper among the available research body, we introduce the main characteristics of the problem studied in this paper:

1) Mobility: The main difference between this paper and the previous work in CCDE [4], [5], [7]-[10] is the notion of mobility. As a direct consequence of mobility, network topology dynamically changes which makes it difficult to find the optimal solution to CCDE. In other words, random moves and limited transmission range of clients would result in variable connectivity degree in different rounds of transmission for different clients. We provide two energy efficient heuristic solutions to this problem.

2) Initial Packet Distribution Variance: The closest work to this paper is [12] where a set of mobile nodes are considered and each client initially owns a packet and wishes to broadcast it to all others. In [12] it has been suggested that in each transmission round all nodes transmit one (network coded) packet which results in large gain over uncoded transmissions. The key difference between our work and [12] is due to the initial variance (imbalance) among the number of missing packets by different clients. In particular, we will show that if the strategy suggested by [12] is used for CCDE, it might be suboptimal in terms of energy efficiency for an arbitrary initial distribution of packets. This is due to the fact some clients with less number of missing packets may obtain the entire set of packets earlier than others and would no longer benefit from transmissions by others. To overcome this issue, we suggest a heuristic in which clients with more knowledge transmit in the initial rounds of transmission to somewhat balance the 
knowledge among clients.

3) Delay Tolerance- Energy Efficiency: In this paper, we mainly focus on the applications where energy consumption should be controlled while delay control is not as important. In such delay tolerant networks, the proposed transmission strategies may suggest a client to wait for better opportunities of transmission to satisfy more clients with any single transmission. Since the topology of the network is time variant, it is likely that such opportunities happen. This is different from the scenario defined in [7] where the main goal is to minimize the delay and the network topology is assumed to be fixed. Similar to [7], we take connectivity degree (the number of neighbors) into account in our formulations, but more conservatively. That is, since the delay is not our main objective and also the clients are mobile, a client can wait for a reasonable time to observe higher connectivity degrees. Recently, delay tolerant ad hoc networks has been the focus of some studies, for instance, [13] propose a network architecture based on opportunistic forwarding using natural human mobility.

To summarize, we will propose two different network coded transmission strategies and will study their total number of transmissions using theoretical and numerical analysis. In the first strategy, all the clients uniformly transmit at each transmission round which is a relatively low delay solution, but not optimal in terms of energy efficiency. In the second strategy, we show that if the clients with both high connectivity degree and knowledge (to be made precise later) are chosen to transmit, the energy consumption is reduced dramatically, but clearly there is a tradeoff between energy and delay. We will provide a parameter in our algorithm to control this tradeoff. The results of numerical experiments verify our analysis. Due to space limitations and obvious gains of network coding over uncoded transmissions in such a dynamic problem, this discussion is not included.

\section{Preliminaries And System Model}

In this section, the notations and problem definition is provided.

Initial Setting: We consider a group of $k$ clients $C=$ $\left\{c_{1}, \ldots, c_{k}\right\}$, all interested in obtaining a set of $n$ packets $X=\left\{x_{1}, \ldots, x_{n}\right\}$. Client $c_{i}$ initially holds an arbitrary subset of $X$, say $X_{i}$. We denote the number of packets client $c_{i}$ initially holds and misses by $n_{i}$ and $\bar{n}_{i}$, respectively. We assume $n_{i}$ (or $\bar{n}_{i}$ ) are random numbers drawn from a probability distribution $p_{N_{i}}\left(n_{i}\right)$ (or $p_{\bar{N}_{i}}\left(\bar{n}_{i}\right)$ ). In this paper, we use a simple packet erasure model to generate the initial distribution of packets, i.e. we assume client $c_{i}$ has received a packet $x_{j} \in X$ with a probability $p_{r}$ independent of other packets, where $p_{r}$ is fixed and identical for all the packets and clients. Therefore $p_{N_{i}}\left(n_{i}\right)$ has a binomial distribution with parameters $\left(n, p_{r}\right)$. However the results are applicable to any other probability distribution.

Mobility and Transmission Model: The clients are initially uniformly distributed over an area $A$. Transmissions are done in several transmission rounds. At each transmission round $t$ each client should make a decision to transmit or not. Each client can transmit at most one packet of length $L$ bits at each round. The algorithm which makes the decision about which clients should transmit at each round is called the transmission strategy. The set of clients that participate in transmission at round $t$ is denoted by $\Omega^{(t)} \subseteq C$.

All the clients have a fixed transmission range $R$. The set of clients located in the transmission range of a client $c_{i}$ at round $t$ are denoted by $\mathcal{N}_{i}^{(t)} \subset C$. If $c_{i} \in \Omega^{(t)}$, a client $c_{j} \in \mathcal{N}_{i}^{(t)}$ will receive a packet transmitted by $c_{i}$ with probability $p_{c}$ and a client $c_{u} \notin \mathcal{N}_{i}^{(t)}$ will not receive a packet transmitted by $c_{i}$. The number of neighbors (or the connectivity degree) of client $c_{i}$ at round $t$ is denoted by $d_{i}^{(t)}=\left|\mathcal{N}_{i}^{(t)}\right|$, which is the realization of a stationary random process $D_{i}^{(t)}$. In other words, we assume $d_{i}^{(t)}$ is an instance of a random variable $D$ with a time invariant probability distribution $p_{D}(d)$. We denote the average connectivity degree by $d_{a v g}=E\{D\}$.

We have used a simple mobility model. At the beginning of each transmission round, a fraction $0 \leqslant \rho \leqslant 1$ of the clients change to new random positions over area $A$. This is consistent with the stationarity of the random processes $D_{i}^{(t)}$ due to [14], where it is shown that the connectivity degree of the nodes in an ad hoc network can be estimated by a binomial distribution with a high accuracy.

Packet Generation and Reservation: The $j$-th unit encoding vector of length $n$ that corresponds to the original packet $x_{j}$ is denoted by $\mathbf{u}_{j}$, where all elements of $\mathbf{u}_{j}$ are zero except the $j$-th element which is 1 . Let us define matrix $\mathbf{K}_{i}^{(t)}$ to represent the knowledge space of a client $c_{i}$ at round $t$. The first $n_{i}$ rows of $\mathbf{K}_{i}^{(t)}$ are vectors $\mathbf{u}_{b}$ of length $n$ for $b$ 's such that $x_{b} \in X_{i}$. Before a client $c_{i}$ receives any information from others, it sends coded packets of the form $\sum_{\ell: x_{\ell} \in X_{i}} \alpha_{\ell} x_{\ell}$, where $\alpha_{\ell}=0$ if $x_{\ell} \notin X_{i}$ and otherwise $\alpha_{\ell} \in \mathbb{F}_{q}$ is the coding coefficient randomly chosen from a finite field with a sufficiently large size $q$. The coding coefficients $\boldsymbol{\alpha}=\left[\alpha_{1}, \cdots, \alpha_{n}\right]$ of any packet received by the client $c_{i}$ are added as the new rows to matrix $\mathbf{K}_{i}^{(t)}$. In general, the coding coefficients of a coded packet generated by the client $c_{i}$ at round $t$ is a linear combination of the rows of $\mathbf{K}_{i}^{(t)}$ with coefficients chosen at random from $\mathbb{F}_{q}$.

$\operatorname{Rank}\left(\mathbf{K}_{i}^{(t)}\right)$ is referred to as the knowledge of client $c_{i}$ at round $t$. Clearly, if the $\operatorname{Rank}\left(\mathbf{K}_{i}^{(t)}\right)$ becomes equal to $n$, then client $c_{i}$ would be able to obtain the entire set of packets. In this paper, since we are trying to give an approximate model, we can assume each network coded packet will increase $\operatorname{Rank}\left(\mathbf{K}_{i}^{(t)}\right)$ by one. This assumption is true with a high probability, but is not discussed here any further.

Objective: In an ideal case, the goal of this paper is to find a transmission strategy that minimizes $\sum_{t=1}^{T}\left|\Omega^{(t)}\right|$ subject to $\operatorname{Rank}\left(\mathbf{K}_{i}^{(T)}\right)=n, \forall c_{i} \in C$. However, finding an exact solution to this problem is difficult or impossible due to the dynamic and random nature of the problem. In this paper, we propose and compare two different transmission strategies and their effect on the total number of transmissions. 


\section{TRAnSmission StRAtegies}

In this paper, we propose and compare two different transmission strategies for the CCDE problem where the clients are assumed to be mobile and the short range links among the clients suffer from packet erasures. The first strategy is conceptually simple where all clients equally participate at each round of transmission. While the procedure is similar to the algorithm studied in [12], we provide a new method to approximate the number of transmissions (in [12] only the order of network coding gain over uncoded transmissions was provided). We call this strategy uniform transmission. However, this approach might be suboptimal due to the initial variance among the number of missing packets by different clients, as some clients may achieve full rank earlier and no longer benefit from others transmissions.

In the second transmission strategy, we propose a heuristic to reduce the total number of transmissions to optimize the total energy consumption. In particular, clients with both largest connectivity degree and rank transmit at each round. Such an opportunistic approach may impose more delay, but since in our problem the main objective is to minimize the energy consumption, the clients can wait for such opportunities. In the rest of this section, we analyze these two strategies and provide some theoretical approximations for the total number of transmissions.

\section{A. Uniform Transmissions}

One straightforward solution to the cooperative data exchange problem for mobile clients is uniform transmission where all the clients transmit at each round. The procedure is represented formally in Algorithm 1.

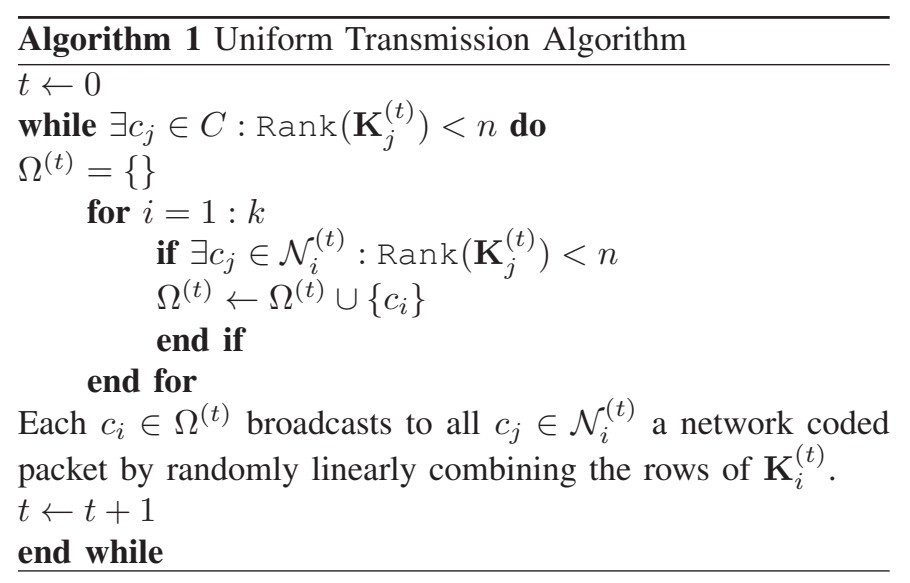

Here, we provide a method to approximate the total number of transmissions required by uniform transmission strategy based on the statistics of the initial distribution of the packets and average connectivity degree. We assume the clients are distributed randomly over the area $A$, therefore the average connectivity degree is $d_{\text {avg }}=\frac{k \pi R^{2}}{A}-1$. The total number of required transmissions can be approximated by Theorem 1 .

Theorem 1. Given the initial probability distribution of the missing packets $p_{\bar{N}_{i}}\left(\bar{n}_{i}\right)$ and cumulative distribution $P_{\bar{N}_{i}}\left(\bar{n}_{i}\right)$, if $M$ is the smallest integer satisfying $P_{\bar{N}_{i}}(M) \geqslant \frac{d_{\text {avg }}}{d_{\text {avg }}+1}$, the total number of transmissions by Algorithm 1 is approximated by $T_{u}=\frac{1}{p_{c}}\left(\frac{M k}{d_{\text {avg }}}+\sum_{i=M+1}^{n} \bar{p}_{\bar{N}_{i}}(i) k(i-M)\right)$.

Although the neighborhood graph of our network is random, in the long run it can be approximated by a $d_{\text {avg-regular }}$ graph. Modeling a random graph with a deterministic graph with a fixed topology is a convenient method to approximate the behavior of our transmission strategy. Inevitably, it cannot be used to determine the actual number of transmissions. However, the gap is shown through numerical experiments to be small. Based on this assumption, the nodes may change their positions but their connectivity degree is fixed. To prove Theorem 1, we break the transmission rounds into two phases. In the first phase, which is called the active phase, all clients participate in transmissions. After a while the majority of clients have achieved full rank and the graph arrives at a point where each client has only one neighbor who still needs to receive more packets. At this point, the second phase, which is called the saturation phase, starts where any single transmission is expected to be useful for only one neighbor. Since the spatial distribution of clients is uniform at each round, we can assume the clients with $\bar{n}_{i} \leqslant M$ missing packets for an appropriately chosen $M$ have achieved full rank at the end of the first phase. Here we have assumed that there is a hard transition between these two phases. But in practice this may not be the case, as a client may find all its neighbors are full rank during the active phase. This simplifying assumption not only eases the calculations, but bypasses the details of events that occur during transmission rounds.

Proof: In the corresponding $d_{\text {avg-regular graph of the }}$ CCDE problem, nodes with full rank are colored in blue and nodes still missing packets are colored in red. Using Lemma 1 (which will be stated immediately after this proof), we know that at the last round of active phase, $\frac{d_{a v g}}{d_{a v g}+1}$ of nodes are blue. Therefore, if $M$ is the smallest integer satisfying $\operatorname{pr}\left(\bar{n}_{i} \leqslant M\right) \geqslant \frac{d_{a v g}}{d_{a v g}+1}$, all clients for which their initial number of missing packets $\bar{n}_{i} \leqslant M$ will be colored in blue in $M$ transmission rounds. Taking connectivity degree and erasure probability into account, during the active phase, a total of $\frac{M k}{p_{c} d_{\text {avg }}}$ transmissions takes place on average.

In the saturation phase, counting the total number of transmissions is equivalent to counting the total number of demands by the clients who still need more packets. Consequently, at this phase it is expected that $\frac{1}{p_{c}} \sum_{i=M+1}^{n} \bar{p}_{\bar{N}_{i}}(i) k(i-M)$ transmissions are required. Theorem 1 follows by adding the number of transmissions during these two phases.

Lemma 1. In a d-regular undirected graph $G(V, E)$ with sets of vertices and edges denoted by $V$ and $E$, respectively, vertices are colored with two colors blue and red such that any blue vertex has only one red neighbor. If the set of blue vertices is denoted by $V_{b}$, then $\frac{\left|V_{b}\right|}{|V|}=\frac{d}{d+1}$.

Proof: Since the graph $G(V, E)$ is $d$-regular it has $\frac{|V| d}{2}$ edges. Since each blue vertex is connected to only one red vertex, if the red vertices and all their connecting edges are 
removed, the remaining graph $G^{\prime}\left(V^{\prime}, E^{\prime}\right)$ would be $(d-1)$ regular graph (each blue vertex has lost one of its edges). Define $\left|V_{r}\right|=|V|-\left|V_{b}\right|$. Hence, $\left|E^{\prime}\right|=|E|-\left|V_{r}\right| d$. Therefore, we have $\frac{\left(|V|-\left|V_{r}\right|\right)(d-1)}{2}=\frac{|V| d}{2}-\left|V_{r}\right| d$ which results in $\frac{|V|-\left|V_{r}\right|}{|V|}=\frac{\left|V_{b}\right|}{|V|}=\frac{d}{d+1}$.

Here, we provide a statistical lower bound for the total number of transmissions in uniform strategy which is given by $\frac{\sum_{i=1}^{n} i p_{\bar{N}_{i}}(i)}{p_{c} d_{\text {avg }}}$. The intuition behind this is straightforward: In the best case scenario, each transmission is beneficial for all the clients receiving that transmission. Since each transmission is expected to satisfy the demand of $d_{\text {avg }} p_{c}$ clients increasing their knowledge by one, the lower bound is given by the dividing the total average number of missing packets $\sum_{i=1}^{n} i p_{\bar{N}_{i}}(i)$ to $d_{a v g} p_{c}$. It should be noted that this bound is statistical and can only explain the expected behavior of the system.

\section{B. Opportunistic Transmissions}

In the second strategy, if a client has the maximum rank among its neighbors and has observed its most connectivity degree among last $W$ transmission rounds, then it is chosen for transmission at that specific round. This will considerably decrease the total number of transmissions at the price of negligible message passing overhead (if the size of packets is sufficiently large). But inevitably, it can impose a large delay specially for a large window size $W$. The procedure is explained formally in Algorithm 2.

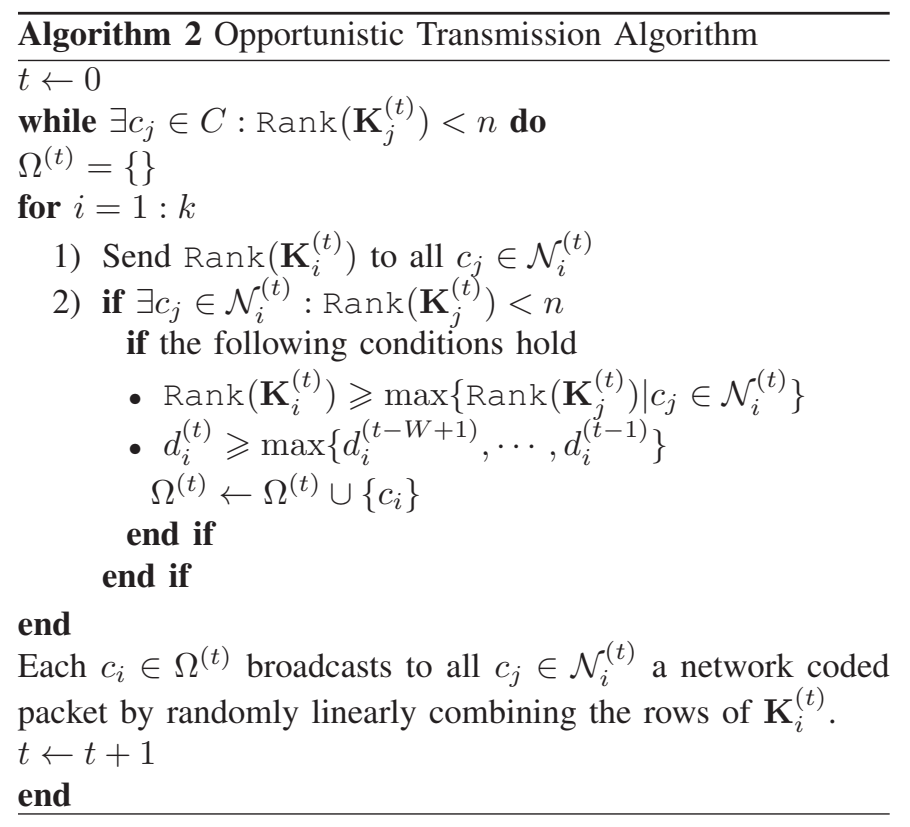

Choosing the clients with higher ranks helps the clients with lower ranks to compensate for their rank deficiency and after that, the rank of all clients grow together. However, while the high rank clients are transmitting, some of their high rank neighbors also benefit and their rank increases. Hence, some of the clients still may achieve full rank earlier than others, which is inevitable. The ideal case can be a basis to give a statistical lower bound for the total number of transmissions, which is the ratio of the total number of missing packets to the average effective connectivity degree. That is,

$$
T_{l o}=\frac{\sum_{i=1}^{n} i p_{\bar{N}_{i}}(i)}{p_{c} d_{e f f}}
$$

The above formulation has an easy interpretation. By each transmission, $d_{\text {eff }}$ neighbors benefit on average. Here, $d_{\text {eff }}$ is expected to be larger than $d_{a v g}$. The reason is that, the algorithm chooses a client if its connectivity degree is the maximum of what it has observed during last $W$ rounds. Therefore, the average of effective connectivity degree is given by:

$$
d_{e f f}=E\left\{\max \left\{D_{i}^{(t-W+1)}, \ldots, D_{i}^{(t)}\right\}\right\}
$$

It is shown in an ad hoc wireless network the connectivity degree of a client, $D_{i}^{(t)}$ can be efficiently estimated by a binomial distribution [14]. In this paper, we use the same model, but we assume there is no shadowing and all the clients have the same transmission range. Based on this assumption the probability distribution of $D_{i}^{(t)}$ would be a binomial distribution with parameters $k-1$ and $\frac{\pi R^{2}}{A}$. If we denote the cumulative distribution of $D_{i}^{(t)}$ by $P_{D}(d)$, then according to [15], [16] the cumulative distribution of $d_{\text {eff }}=\max \left\{D_{i}^{(t-W+1)}, \ldots, D_{i}^{(t)}\right\}$ can be easily calculated by:

$$
P_{D_{e f f}}\left(d_{e f f}\right)=\left(P_{D}(d)\right)^{W}
$$

and the expectation would be derived easily. It should be mentioned the $d_{e f f}$ is an increasing function of $W$. This is expected since as $W$ gets larger, the chance of observing larger connectivity degrees would be higher and asymptotically we have:

$$
\lim _{W \rightarrow+\infty} d_{e f f}=k-1
$$

In practice, $W$ is a parameter to control the delay. In the numerical section, the delay-energy tradeoff will be studied through simulations.

\section{NuMERICAL RESUlTS}

In this section, we provide the numerical results to verify our analysis, evaluate the performance of the proposed strategies and demonstrate the delay-energy tradeoff in the opportunistic strategy. The simulations are done over a square area of $100 \mathrm{~m} \times 100 \mathrm{~m}$. The transmission range of each client is assumed to be $R=30 \mathrm{~m}$. We have $k=40$ clients and each client initially has received each packet $x_{j}$ with a variable probability $p_{r}$ from a set of $n=50$ packets. We have set the mobility parameter $\rho=1$ and $p_{c}=0.9$.

Fig. 1 compares the total number of transmissions for the uniform and the opportunistic strategies and compares these results with analytical calculations. Two statistical lower bounds for the mentioned strategies are provided as well as the approximation for total number of transmissions in uniform strategy. As it can be inferred from the figure, the difference between this approximation and simulation results is very small especially when compared to the difference between 


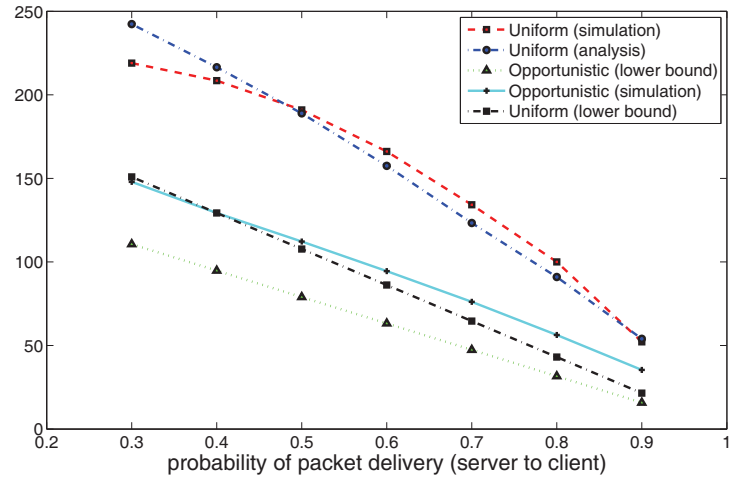

Fig. 1. Total number of transmissions for the proposed strategies in theory and simulations

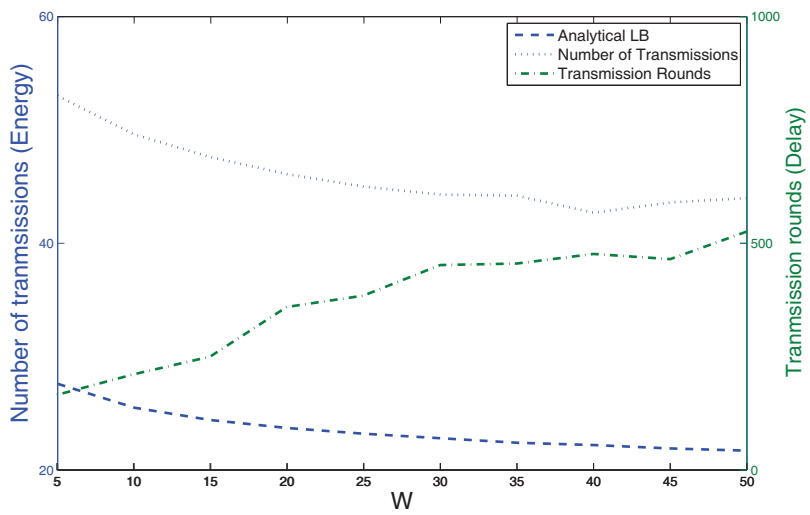

Fig. 2. Energy-delay tradeoff: total number of transmission vs. total transmission rounds in opportunistic strategy for different values of $W$

the lower bound and simulation results. The values are the averages of 50 trials for each value of $p_{r}=0.3,0.4, \ldots, 0.9$. This numerical error is not only produced by the approximation algorithm but may also be a result of corner effect in the square area used here.

The trade off between energy and delay is evaluated in Fig. 2 for $p_{r}=0.85$ for different values of $W$ ranging from 5 to 50 . The total number of transmission rounds is used as a metric to measure the delay. Also, the total number of transmissions is considered to be equivalent to the total energy consumption of both strategies. We can see that at $W=10$, the number of rounds is 211 and the average number of simulated transmissions is 49.6. Therefore, the total number of messages exchanged to inform the ranks of clients is $211 \times 40$ for $k=40$ clients. If the length of a rank information message is $10^{-4}$ times of the length of a packet, then the total amount of overhead imposed by these message passings is less than the length of one packet which is around $2 \%$ of the total amount of transmissions.

\section{CONCLUSiON}

In this paper, we compared two different network coded transmission strategies for the cooperative data exchange problem. We showed if the clients with high rank and high connectivity degree transmit at each round, the total number of transmissions is considerably reduced. This would be at the price of more delay to wait for better opportunities for transmission. Providing a theoretical model for this delay is an interesting direction for future work. Also considering more realistic mobility model and its impact on the energy consumption and delay would be another worthwhile topic to consider.

\section{ACKNOWLEDGMENT}

This work was supported under Australian Research Council Discovery Projects funding scheme (project no. DP0984950).

\section{REFERENCES}

[1] A. Nosratinia, T. E. Hunter, and A. Hedayat, "Cooperative coommunications in wireless networks," IEEE Communications Magazine, vol. 42, pp. 74-80, 2004.

[2] F. Fitzek and M. Katz, Cooperation in Wireless Communications: Principles and Applications. Springer, 2007.

[3] Q. Lv, P. Cao, E. Cohen, K. Li, and S. Shenker, "Search and replication in unstructured peer-to-peer networks," in Proc. 16th international conference on Supercomputing (ICS), New York, USA, 2002, pp. 84-95.

[4] S. E. Rouayheb, A. Sprinston, and P. Sadeghi, "On coding for cooperative data exchange," in Proc. IEEE Inf.. Theory Workshop (ITW), Cairo, Egypt, Jan. 2010, pp. 118-122.

[5] A. Sprinston, P. Sadeghi, G. Booker, and S. E. Rouayheb, "A randomized algorithm and performance bounds for coded cooeprative data exchange," in Proc. IEEE Int. Symp. Inf. Theory (ISIT), Austin, TX, Jun. 2010, pp. 1888-1892.

[6] R. Ahlswede, N. Cai, S.-Y. R. Li, and R. Yeung, "Nework information flow," IEEE Trans. Inf. Theory, vol. 46, pp. 1204-1216, July 2000.

[7] X. Liu, S. Raza, C. N. Chuah, and G. Cheung, "Network coding based cooperative peer-to-peer repair in wireless ad-hoc networks," in IEEE International Conference of Communication (ICC), Beijing, China, May. 2008.

[8] S. E. Tajbakhsh and P. Sadeghi, "A generalized model for cost and fairness analysis in coded cooperative data exchange," in The 2011 International Symposium on Network Coding (Netcod), Beijing, China, July. 2011.

[9] T. Courtade, B. Xie, and R. Wesel, "Optimal exchange of packets for universal recovery in broadcast networks," in Proc. IEEE Military Communications Conference (MILCOM), San Jose, CA, Nov. 2010.

[10] D. Ozgul and A. Sprinston, "An algorithm for cooperative data exchange with cost criterion," in Information Theory and Applications Workshop (ITA), San Diego, CA, Feb. 2011.

[11] T. Ho, M. Medard, J. Shi, M. Effros, and D. R. Karger, "On randomized network coding," in In Proceedings of 41st Annual Allerton Conference on Communication, Control, and Computing, 2003.

[12] C. Fragouli, J. Widmer, and J. Y. le Boudec, "On the benefits of network coding for wireless applications," in In Proc. Fourth International Symposium on Modeling and Optimization in Mobile, Ad Hoc and Wireless Networks, Boston, MA, Apr. 2006.

[13] J. Su, J. Scott, P. Hui, J. Crowcroft, E. de Lara, C. Diot, A. Goel, M. H. Lim, and E. Upton, "Haggle: Seamless networking for mobile applications," in In Proc. Ubiquitous Computing, Inssbruck, Austria, Sep. 2007.

[14] R. Hekmat and P. V. Mieghem, "Degree distribution and hopcount in wireless ad-hoc networks," in In Proc. The IEEE International Conference on Networks (ICON2003), Sydney, Australia, Sep. 2003.

[15] A. Papoulis and S. U. Pillai, Probability, Random Variables and Stochastic Processes. New York, NY: McGraw-Hill Higher Education, 2002.

[16] M. N. Tata, "On outstanding values in a sequence of random variables," Probability Theory and Related Fields, vol. 12, pp. 9-20, 1969. 